\title{
Preparing for the next pandemic
}

\author{
The COVID-19 pandemic has opened a window of opportunity for rethinking the way countries prepare for \\ public-health crises. This window must not be wasted.
}

1 year has gone by since the World Health Organization declared the COVID-19 pandemic a global pandemic on 11 March 2020. Nearly 100 million people have become sick worldwide, more than 2 million have died, and many survivors are experiencing long-haul symptoms. Despite advances in the vaccination and clinical management of patients with COVID-19, the end of the worst public-health crisis in a century is not nearly in sight yet. In this issue, we launch a special Focus dedicated to understanding the long-term impact of the COVID-19 pandemic and to begin to explore the lessons that can be learned from the mistakes made in handling the current health crisis, in order to prepare for future crises.

In the course of preparing this special issue of Nature Medicine, we reached out to many experts who have been involved in the response to the COVID-19 pandemic, including researchers who have contributed seminal research that allowed understanding of the biology of SARS-CoV-2, the host immune response to the virus and how COVID-19 manifests clinically, to test the efficacy of therapeutic approaches and to develop vaccines. We also spoke with epidemiologists, public-health experts, policymakers, and researchers whose work focuses on the resilience of health systems, governance and the implementation of health policies. From these conversationscaptured in pieces published in this issue and others that will appear throughout the year in our pages-a few common themes emerged.

On the one hand, there is a sense of scientific accomplishment, and that the global research community has collectively risen to the challenge imposed by the pandemic. In no other time in the history of science has so much information been generated, shared and deployed so quickly to address a threat to humanity. On the other hand is the realization that no amount of research and scientific development can compensate for ineffective leadership and a lack of coordination within governments and international cooperation between countries. More than anything else, the COVID-19 crisis has been a failure of governance.

There is a clear sense that preparing for the next pandemic will require a deep dive into those failures, understanding what went wrong and working toward improving the architecture of health-security governance, a point made by John Nkengasong.

Overcoming institutional complacency is also critical to the development of a long-term pandemic preparedness strategy that is driven by scientific evidence and education of the population and that strives for health security, as argued by former World Health Organization Director-General Margaret Chan. It is a tragic lesson of the COVID-19 pandemic that its death toll is amplified by myriad underlying common health conditions that are increasingly more prevalent in the general population, such as diabetes, obesity and hypertension. Increasing the resilience of health systems through universal healthcare policies can improve the overall health of populations and may reduce the burden of fatalities in future outbreaks.

Achieving universal healthcare is not without its challenges, but it is possible, and Ole Norheim and colleagues draw a map of how packages of essential health services can be developed in resource-constrained settings, drawing on their experience developing such packages in Ethiopia. Paul Spiegel further describes the challenges of responding to a pandemic in humanitarian settings, and Devi Sridhar and colleagues sum up the current state of affairs in global health governance in this first year of the pandemic, with an eye on lessons for the future. Madhukar Pai and colleagues tackle an often overlooked topic that has proven to be a major bottleneck in the public-health response during the current pandemic: the problem of limited diagnostic testing capacity, and the need to build a better structure for testing as part of pandemic preparedness strategies.

Governance failure has also manifested through a proliferation of misinformation and distrust in science and health professionals and a subsequent lack of compliance with public-health measures that can save lives and curtail a pandemic. Elizabeth Higgs and colleagues present an overview of good practices that enhance public engagement in research and with medicine during an ongoing public-health crisis. Imran Ahmed discusses the challenges of dealing with an increasing resistance to vaccination and the need to understand what is behind the misinformation industry. The importance of acquiring reliable information during an ongoing public-health crisis and how such data can be used to feed and build models to aid in the understanding of key pathogenic, epidemiologic, clinical and socio-behavioral characteristics of an ongoing outbreak are covered in a Perspective by Joseph Wu and colleagues. Speaking of predicting future threats, Mike May spoke with several infectious-disease experts about what tomorrow's biggest microbial threats could be. And Ralph Baric and Rita Meganck present an overview of the drug-development pipeline for new infectious agents, and the need for new vision, dedicated funding and private, federal and academic partnership models.

Finally, thinking of how the pandemic has affected our community of researchers, clinicians and health professionals, we have asked our readers to share with us how they have used art to document their experiences through the past year and to cope with the uncertainties imposed by the pandemic. We received many more submissions on Twitter then we could ever highlight in a single issue, and a selection, chosen by the Nature Medicine team, is presented in a special feature and on the cover of this issue of the journal.

The world is still pretty much in the midst of a crisis, and one might argue that is too early to start talking of preparing for future pandemics. We disagree-we think that now is the right time to talk about the future and about the changes that need to happen to improve the global capacity to respond to health crises of this magnitude. Experience from the recent past has repeatedly shown that resources, funding and political capital tend to taper off as an outbreak does. The true impact of COVID-19, however, in both health terms and economic terms, is likely to outlast the duration of the acute phase of the pandemic, opening an window of opportunity for enduring change while the virus is still on people's minds. This window of opportunity must not be wasted.

Published online: 15 March 2021

https://doi.org/10.1038/s41591-021-01291-Z 